Article

\title{
Effect of Temperature and Texture on Hall-Petch Strengthening by Grain and Annealing Twin Boundaries in the MnFeNi Medium-Entropy Alloy
}

\author{
Mike Schneider*(D), Felicitas Werner, Dennis Langenkämper, Christian Reinhart and \\ Guillaume Laplanche ${ }^{\mathbb{D}}$ \\ Institut für Werkstoffe, Ruhr-Universität Bochum, D-44780 Bochum, Germany; felicitas.werner@rub.de (F.W.); \\ dennis.langenkaemper@rub.de (D.L.); christian.reinhart@rub.de (C.R.); guillaume.laplanche@rub.de (G.L.) \\ * Correspondence: mike.schneider@rub.de; Tel.: +49-234-32-27349
}

Received: 21 December 2018; Accepted: 11 January 2019; Published: 15 January 2019

\begin{abstract}
Among equiatomic alloys of the $\mathrm{Cr}-\mathrm{Mn}-\mathrm{Fe}-\mathrm{Co}-\mathrm{Ni}$ system, MnFeNi was shown to exhibit a strong anti-invar behavior but little is known regarding its mechanical properties. The objective of the present study is to investigate Hall-Petch strengthening by grain and annealing twin boundaries in MnFeNi. For this purpose, seven different grain sizes between 17 and $216 \mu \mathrm{m}$ were produced. Mean grain sizes (excluding annealing twin boundaries) and crystallite sizes (including them) were determined using the linear intercept method. Overall, $25 \%$ of the boundaries were found to be annealing twin boundaries regardless of the grain size. In some cases, two twin boundaries can be present in one grain forming an annealing twin, which thickness represents one quarter of the mean grain size. Based on a comparison of the mean twin thickness of different alloys with different stacking fault energy (SFE), we estimated an SFE of $80 \pm 20 \mathrm{~mJ} / \mathrm{m}^{2}$ for MnFeNi. Compression tests of MnFeNi with different grain sizes were performed between 77 and $873 \mathrm{~K}$ and revealed a parallel shift of the Hall-Petch lines with temperature. The interaction between dislocations and boundaries was investigated by scanning transmission electron microscopy (STEM) in a deformed specimen. It was found that a large number of dislocations are piling up against grain boundaries while the pile-ups at annealing twin boundaries contain much fewer dislocations. This indicates that annealing twin boundaries in this alloy are less effective obstacles to dislocation motion than grain boundaries.
\end{abstract}

Keywords: high-entropy alloys; CoCrFeMnNi; FeNiMn; hall-petch effect; dislocations/grain boundaries interactions; texture; mechanical properties

\section{Introduction}

Yeh et al. [1] first introduced the concept of high-entropy alloys (HEAs) which represent a new class of metallic materials composed of at least five elements in approximately equiatomic concentrations. Later on, Yeh et al. [2] further classified alloys comprising two to four elements in near equiatomic proportions as medium-entropy alloys (MEAs). Several of these alloys may form disordered solid solutions with simple crystallographic structures, e.g., face centered cubic (FCC) $[3,4]$ and body centered cubic (BCC) [5-7]. Equiatomic FCC alloys from the Cr-Mn-Fe-Co-Ni system have attracted a lot of interest in the scientific community due to their very interesting mechanical properties, namely, a good combination of ductility and ultimate tensile strength [8,9], a high work-hardening rate $[10,11]$, an impressive fracture toughness [12], and an excellent resistance to fatigue-crack growth [13]. Wu et al. [14] were the first to investigate the phase stability and mechanical properties of several equiatomic MEAs of the $\mathrm{Cr}-\mathrm{Mn}-\mathrm{Fe}-\mathrm{Co}-\mathrm{Ni}$ system. Most of these MEAs were found to be single phase FCC after casting and homogenization as well as after cold rolling and recrystallization [14]. 
Moreover, Wu et al. [14] observed that the MEAs have different densities of annealing twins in the recrystallized state with $\mathrm{CrCoNi}$ and $\mathrm{CrFeCoNi}$ showing the highest densities while $\mathrm{MnFeNi}, \mathrm{FeNi}$, and pure Ni have the lowest. The authors suggested that this trend may reflect the evolution of stacking fault energy (SFE) with composition [14], i.e., the higher the SFE the lower the annealing twin density. Later on, Laplanche et al. [15] studied the evolution of physical properties with temperature of a set of MEAs including magnetic and elastic properties as well as thermal dilatation. Interestingly they found that the $\mathrm{MnFeNi}$ alloy is paramagnetic above $77 \mathrm{~K}$, shows the largest coefficient of thermal expansion of the investigated MEAs, and exhibits an anti-invar behavior [15], (i.e., an enhanced coefficient of thermal expansion $[16,17])$. This exceptional physical property makes the MnFeNi alloy very attractive for potential applications as bimetallic strips in which a high thermal dilation and high strength are required.

While the temperature dependence of the tensile properties of $\mathrm{MnFeNi}$ with a mean grain size of $\sim 30 \mu \mathrm{m}$ was already reported [9], to our knowledge an examination of the grain size dependence of the yield stress (Hall-Petch relationship) and the effect of temperature on intrinsic lattice strength for this alloy has never been reported. The only studies regarding annealing twin boundary strengthening in the Cr-Mn-Fe-Co-Ni system focused on equiatomic CrMnFeCoNi [10,18,19], CrFeCoNi [9], and $\mathrm{CrCoNi}$ at room temperature $[20,21]$. Further studies concerning strengthening due to deformation twins in CrMnFeCoNi have been reported in [11,22,23]. In the most recent study, Schneider et al. [21] suggested that the strength of annealing twin boundaries is related to the stress required to constrict Shockley partials into a perfect dislocation. Based on this suggestion, it is expected that the strength of annealing twin boundaries decreases with increasing SFE, i.e., annealing twin boundaries in $\mathrm{MnFeNi}$ should be weaker than in other alloys such as $\mathrm{CrCoNi}$ and $\mathrm{CrMnFeCoNi}$.

In the present study, compression tests were carried out between 77 and $873 \mathrm{~K}$ on the ternary equiatomic MnFeNi alloy with seven different grain sizes ranging from 17 to $216 \mu \mathrm{m}$. Grain size and texture analyses were carried out using backscattered electron imaging (BSE) and electron backscatter diffraction (EBSD), respectively. The effect of temperature and texture on grain size hardening (Hall-Petch relationship) and intrinsic lattice strength are investigated.

\section{Experimental Methods}

A cylindrical $\mathrm{Mn}_{33.3} \mathrm{Fe}_{33.3} \mathrm{Ni}_{33.3}$ (in at.\%) ingot with a weight of $2.1 \mathrm{~kg}$, a diameter of $45 \mathrm{~mm}$, and a height of $140 \mathrm{~mm}$ was produced. For this purpose, pure elements (purity $>99.9 \mathrm{wt} . \%$, Hauner Metallische Werkstoffe $\mathrm{GmbH}$, Röttenbach, Germany) were vacuum induction melted in a high purity argon atmosphere (99.998 vol.\%) in a furnace of type Leybold Heraeus IS 1/III. Prior to melting, pure $\mathrm{Mn}$ flakes were etched to remove their oxide layer using the following procedure: (i) $700 \mathrm{~g}$ of Mn were immersed in $500 \mathrm{~mL}$ of deionized water, (ii) a solution of nitric acid (38 vol.\% in distilled water) was then added stepwise under constant manual stirring until a shiny surface was visible (overall $\sim 100 \mathrm{~mL}$ were added in five steps), (iii) $500 \mathrm{~mL}$ of deionized water were subsequently added to dilute and neutralize the acid, and (iv) Mn was further rinsed with ethanol and dried using a hair dryer. After casting, the diameter of the cylindrical ingots was reduced from 45 to $40 \mathrm{~mm}$ by turning it on a lathe. The ingot was then cut perpendicular to the cylindrical axis into halves, which were sealed independently in evacuated quartz tubes, homogenized at $1373 \mathrm{~K}$ for $48 \mathrm{~h}$, air-cooled to room temperature (RT), and removed from the quartz tubes. The diameter of the two cylinders was reduced from 40 to $8.1 \mathrm{~mm}$ in 11 steps (true strain $~ 3.2$ ) using a rotary swaging machine of type HMP R6-4-120-21S (HMP Umformtechnik GmbH, Pforzheim, Germany). In a previous study, the chemical composition of the same swaged rod was determined by $\mathrm{X}$-ray fluorescence analysis and found to be $\mathrm{Mn}_{33.1} \mathrm{Fe}_{34.3} \mathrm{Ni}_{32.6}$, which is very close to the nominal composition [15]. Besides, since the concentration of impurities such as C (0.024 at.\%), O (0.033 at.\%), and S (0.016 at.\%) are relatively low, it is therefore reasonable to neglect the effect of these interstitials on mechanical properties.

From the swaged rods (diameter: $8.1 \mathrm{~mm}$ ), 45 compression specimens with dimensions: $4 \mathrm{~mm}$ diameter and $8 \mathrm{~mm}$ height were machined on a lathe. Afterwards, the faces of the compression 
specimen were ground using a corundum grinding stone (particle size $\sim 250 \mu \mathrm{m}$ ) to achieve a parallelism better than $1^{\circ}$. As MEAs and HEAs among the $\mathrm{Cr}-\mathrm{Mn}-\mathrm{Fe}-\mathrm{Co}-\mathrm{Ni}$ system which contain $\mathrm{Mn}$ were reported in the literature to easily oxidize in air above $600{ }^{\circ} \mathrm{C}$ [24-26], all recrystallization heat treatments performed in the present study were carried out in evacuated quartz tubes. Even though Mn has a low vapor pressure, energy-dispersive X-ray spectroscopy (EDX) analyses (not shown here) did not reveal any significant Mn losses after annealing at $1473 \mathrm{~K}$ for $1 \mathrm{~h}$. To minimize the potential for scatter, five compression samples and a metallographic specimen were annealed at the same time, see Figure 1, at temperatures between 1073 and $1473 \mathrm{~K}$ for times ranging from $45 \mathrm{~min}$ to $2 \mathrm{~h}$ to establish seven different grain sizes. After the heat treatments, the samples were air-cooled and removed from the quartz tubes. All metallographic samples were embedded in epoxy, ground with SiC abrasive paper down to a grain size of $\sim 7 \mu \mathrm{m}$, polished with diamond suspensions to $1 \mu \mathrm{m}$, and finally vibropolished for $48 \mathrm{~h}$ (Vibromet 2, Buehler, Esslingen, Germany) in colloidal silica having a particle size of $0.06 \mu \mathrm{m}$.

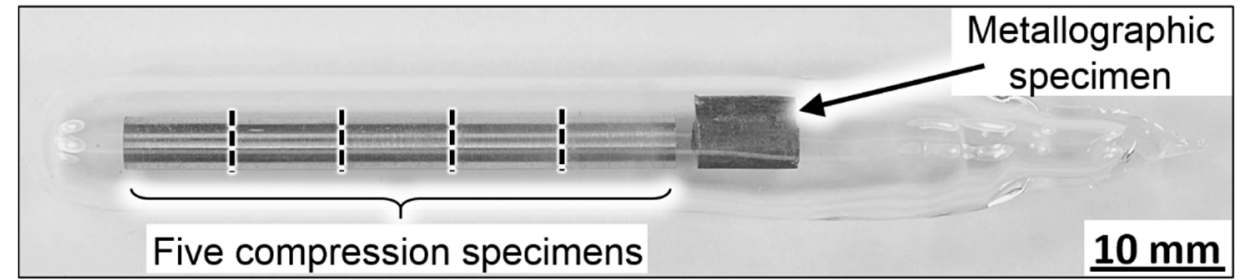

Figure 1. Photograph of five compression specimens and one metallographic sample encapsulated in an evacuated quartz tube for subsequent heat treatment.

To investigate the interaction between dislocations and grain and annealing twin boundaries, slices for scanning transmission electron microscopy (STEM) analyses were cut from compression specimens deformed to $1.5 \%$ plastic strain using a low-speed cutting machine (IsoMet, Buehler, Esslingen, Germany). These slices were cut at $45^{\circ}$ from the compression axis, ground to a thickness of $100 \mu \mathrm{m}$ using 1000-grit SiC paper, and TEM foils were then prepared at $253 \mathrm{~K}$ by double-jet electrochemical thinning in a TenuPol-5 (Struers, Willich, Germany) operating at 18 V. The electrolyte consisted of $70 \mathrm{vol} \% \%$ methanol, $20 \mathrm{vol}$ \% glycerine, and $10 \mathrm{vol} \% \%$ perchloric acid. STEM analyses were conducted in a Tecnai F20 Super-Twin (FEI, Hillsbro, OR, USA) operating at $200 \mathrm{kV}$, equipped with a scanning unit.

Before we show the methods used to characterize the microstructure of the recrystallized specimens, we review three key issues in scientific reports which make an accurate comparison of grain size data reported in literature difficult: (1) There are two definitions of the grain size which are commonly used in the scientific community. The mean grain size is often defined as the mean intercept distance $\bar{\ell}$ or as the volumetric grain size $\bar{D}=1.571 \bar{\ell}[27,28]$. However, researchers rarely clearly state the definition they used which brings confusion when comparing grain sizes obtained by different groups. (2) Even if it is often claimed that the ASTM E-112 standard method was followed, the "experimental methods" section rarely reports mandatory information such as the number of fields measured, the magnification of these, and the number of intersections counted (see Section 18 in Ref. [28]). More importantly the confidence interval of the mean grain size is rarely reported. Therefore, it is often unclear whether the reported grain sizes were obtained from a statistically significant number of measurements. (3) There is currently no consensus in the scientific community on whether annealing twin boundaries $(\Sigma 3)$ in FCC materials should be counted as barriers to dislocation motion or not. In the literature, the assessment of grain size strengthening in FCC alloys (Hall-Petch parameters) can be divided into two groups: First, those (including the ASTM E-112) which assume that annealing twin boundaries are weak barriers to dislocation motion and should be excluded from the grain size analysis, e.g., [10,29-31]. Second, a group for which the annealing twin boundaries are as strong as or even stronger than high angle grain boundaries, e.g., [19,32-37]. Since grain sizes are not systematically assessed in the same way, i.e., annealing twin boundaries are either counted or excluded from the 
grain size analysis, this makes a comparison of grain sizes and Hall-Petch parameters reported by different groups difficult.

In the present paper, we are interested in boundary strengthening and we think that the mean intercept distance $\bar{\ell}$ provides a better representation of the mean free path of dislocations, since dislocation sources are randomly distributed within grains. Therefore, in the present paper we use the mean intercept distance and for the sake of simplicity we refer to it as the mean grain size in the remainder of the paper. To not lose any information regarding the microstructures of the polycrystalline MnFeNi alloys, an effort was made to assess the mean grain size (d) excluding annealing twin boundaries, the mean crystallite size $(c)$ accounting for both grain and twin boundaries, the average thickness of annealing twins $(t)$ and the average number of twin boundaries per grain $(n)$ defined as $n=(-1+d / c)$. For grain size analysis, four backscatter electron (BSE) micrographs with a resolution of $4096 \times 3775$ pixels were taken at locations spaced $1 \mathrm{~mm}$ apart between the center and the outer surface of the recrystallized metallographic specimens. For this purpose, a scanning electron microscope (SEM) of type Quanta 650 ESEM (FEI, Hillsbro, OR, USA) was used. Depending on the mean grain size, accelerating voltages ranging from 10 (small grain size) to $20 \mathrm{kV}$ (large grain size) were applied at a working distance of $10 \mathrm{~mm}$ to optimize the BSE contrast. Mean grain sizes were determined using the Heyn Lineal Intercept procedure highlighted in Section 13 of the standard test method ASTM E-112 [28]. Four horizontal equidistant and parallel lines of identical length as well as four vertical lines resulting in 300 to 500 intercepts per micrograph were used, see Figure 2 . In the present paper, the mean grain size is calculated as the average of four independent measurements and a relative accuracy of $6 \%$ was obtained with $~ 1600$ intercepts per sample. Thus, the mean grain sizes and corresponding standard deviations given in Table 1 are statistically representative of the recrystallized microstructures.

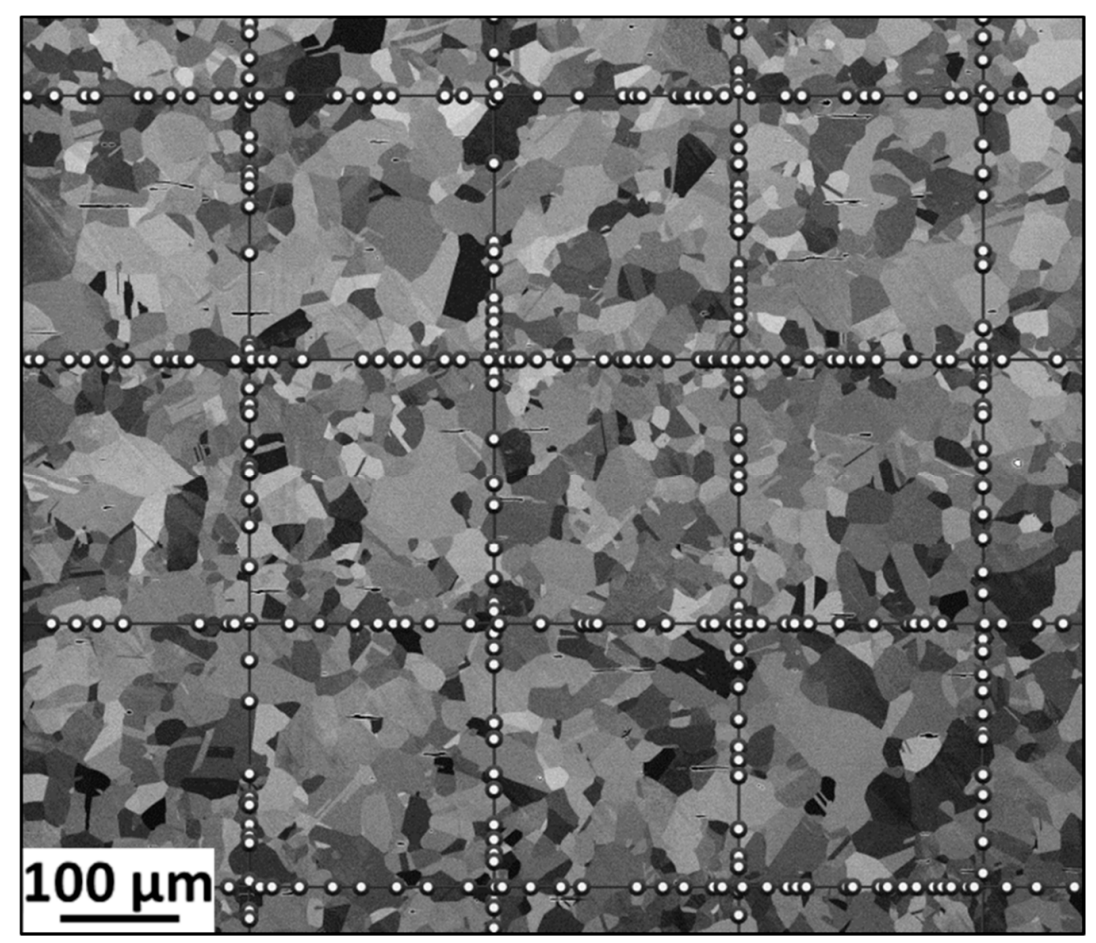

Figure 2. Representative backscatter electron (BSE) micrograph of a recrystallized microstructure $(d=19 \mu \mathrm{m}, c=16 \mu \mathrm{m})$ with four horizontal and four vertical lines used for grain size analysis using the Heyn lineal intercept procedure.

The texture of the recrystallized samples was determined by EBSD using an acceleration voltage of $30 \mathrm{kV}$, a working distance between 12 and $17 \mathrm{~mm}$ and step sizes between 0.3 and $3 \mu \mathrm{m}$. All measurements were conducted using the above-mentioned SEM equipped with a Hikari XP camera 
(EDAX Inc., Mahwah, NJ, USA). Indexing of the Kikuchi patterns was performed using the software TSL OIM Analysis (version 6.2.0, EDAX Inc., Mahwah, NJ, USA).

Table 1. Mean grain size $(d)$, crystallite size $(c)$ and number of annealing twin boundaries per grain (n) after heat treatment at different temperatures and times. $d$ only takes into account the intercepts with grain boundaries, whereas $c$ is determined by counting intercepts with both grain and annealing twin boundaries.

\begin{tabular}{ccccccc}
\hline $\boldsymbol{T}(\mathbf{K})$ & Time $(\mathbf{m i n})$ & $\boldsymbol{d}(\boldsymbol{\mu m})$ & $\boldsymbol{c}(\boldsymbol{\mu m})$ & $\boldsymbol{n ( - )}$ & $\boldsymbol{t}(\boldsymbol{\mu m})$ & Magnification \\
\hline 1073 & 45 & $17 \pm 1$ & $13 \pm 1$ & 0.3 & 4.5 & 250 \\
1073 & 60 & $19 \pm 1$ & $16 \pm 1$ & 0.1 & 7.0 & 150 \\
1073 & 120 & $22 \pm 2$ & $14 \pm 1$ & 0.6 & 7.0 & 200 \\
1173 & 60 & $33 \pm 2$ & $25 \pm 1$ & 0.3 & 15 & 100 \\
1273 & 60 & $66 \pm 2$ & $49 \pm 4$ & 0.3 & 21 & 75 \\
1373 & 30 & $112 \pm 5$ & $86 \pm 6$ & 0.3 & 30 & 75 \\
1473 & 60 & $216 \pm 10$ & $120 \pm 8$ & 0.8 & 51 & 70 \\
\hline
\end{tabular}

All compression tests were performed using a XForce Z100 machine (Zwick Roell GmbH \& Co KG, Ulm, Germany) at temperatures ranging from 77 to $873 \mathrm{~K}$ and at a nominal strain-rate of $10^{-3} \mathrm{~s}^{-1}$. Tests performed in the temperature range (173-873 K) were performed in a temperature-controlled chamber in air. The temperature was held for $45 \mathrm{~min}$ before testing to ensure thermal equilibrium. Tests performed at $77 \mathrm{~K}$ were conducted in liquid nitrogen. The samples and compression plates were completely covered with liquid nitrogen for $15 \mathrm{~min}$ prior to and during testing.

\section{Results}

\subsection{Grain and Crystallite Sizes}

Seven different grain sizes $(d)$, given in Table 1 , were established using the heat treatments presented in Section 2. Additionally, Table 1 lists the corresponding annealing temperatures $(T)$ and times, the mean crystallite size $(c)$, the number of annealing twin boundaries per grain $(n)$, the average twin thickness $(t)$, and the magnification of the BSE micrographs used to determine grain sizes.

Figure 3 shows microstructural features associated with annealing twin boundaries. The number of annealing twin boundaries per grain $(n)$ and the average thickness of annealing twins $(t)$ are plotted as a function of the mean grain size $(d)$ in Figure $3 a, b$, respectively. Despite some scatter, $n$ is roughly constant and equal to $0.35 \pm 0.1$ for all grain sizes (Figure 3a) while $t$ increases linearly with $d$ with a slope of $0.25 \pm 0.2$. In other words, there are two twin boundaries in one out of six grains and the annealing twin thickness is roughly one quarter of the mean grain size.
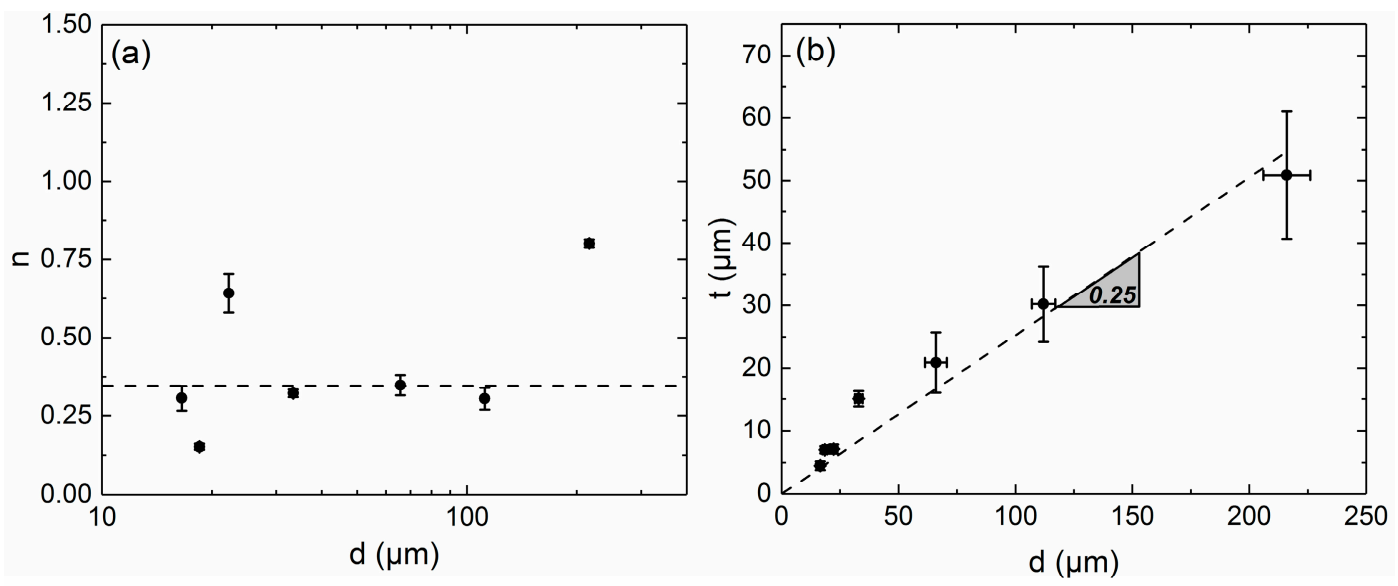

Figure 3. (a) Number $(n)$ of annealing twin boundaries per grain and (b) average twin thickness $(t)$ as a function of the average grain size $(d)$. 


\subsection{Texture}

Texture analyses of recrystallized MnFeNi for (a) the smallest, (b) a medium, and (c) the largest crystallite size are presented in Figure 4. The first column shows BSE images with the same magnification, the second orientation maps where crystallites are colored according to their crystallographic orientation relative to the longitudinal axis of the swaged rods (indicated by a white arrow in the lower right of the BSE image in Figure 4a), and the third inverse pole figures (IPFs) for which the scale in multiple times random is given in the upper right corner of Figure 4. It can be seen from the crystallite orientation maps and IPFs that no strong texture is present. For the largest crystallite size, three scans taken at locations spaced $2 \mathrm{~mm}$ apart were recorded to average the texture over a greater number of grains than only those shown in the crystallite orientation map in Figure 4c. As these three scans yielded similar results, the IPF for the largest crystallite size is therefore representative of the microstructure (see Figure 4c). Using EBSD, Taylor factors $(M)$ were calculated for all crystallite sizes, which were found to be very close to 3.06 (maximum deviation: $3 \%$ ), regardless of the mean grain size. This $M$-value is representative of an isotropic polycrystal (without texture). Therefore, we conclude that texture does not affect the mechanical properties reported in the present study.
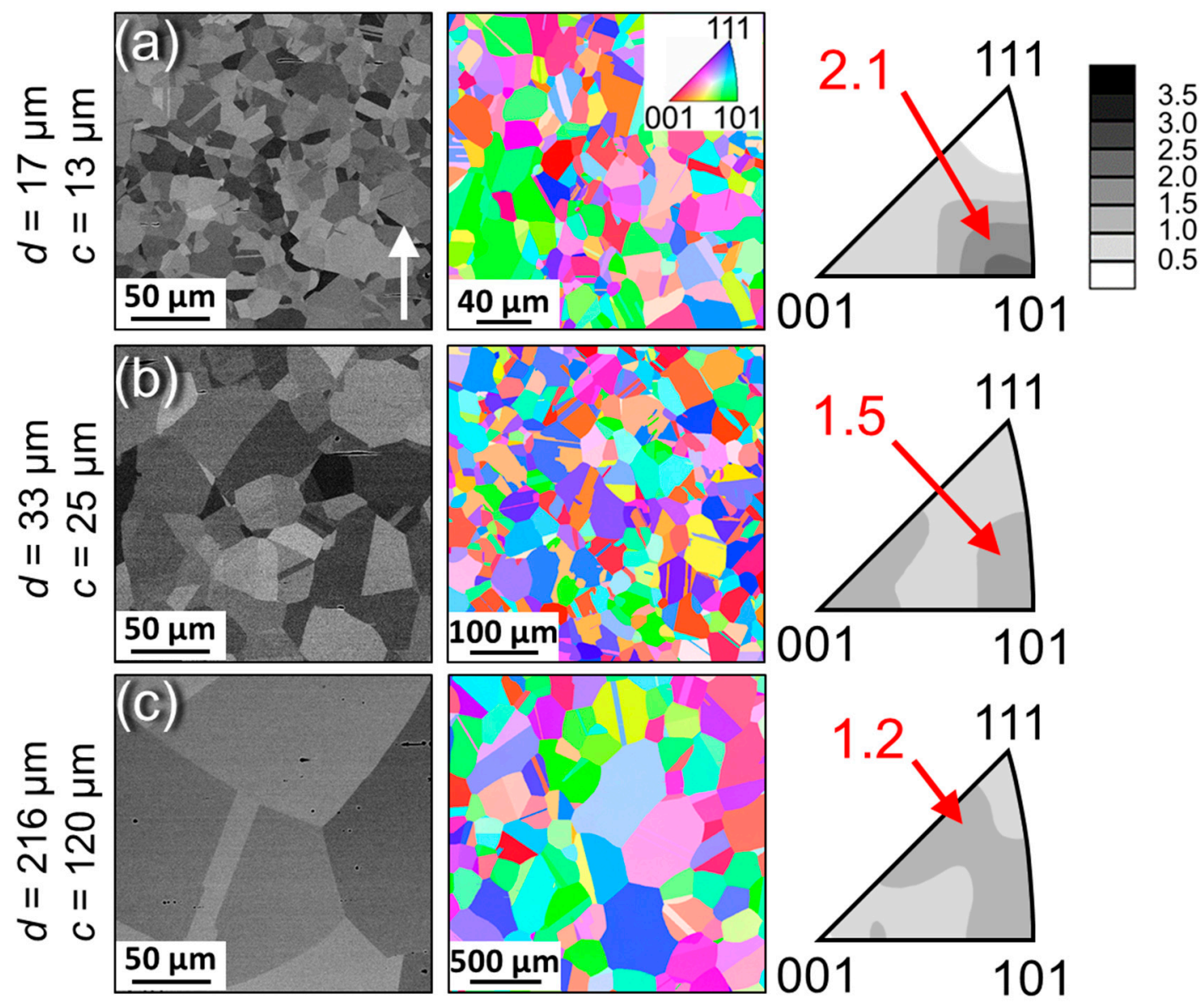

Figure 4. Representative microstructures of recrystallized MnFeNi with different grain and crystallite sizes: (a) $d=17 \mu \mathrm{m}, c=13 \mu \mathrm{m},(\mathbf{b}) d=33 \mu \mathrm{m}, c=25 \mu \mathrm{m}$, (c) $d=216 \mu \mathrm{m}, c=120 \mu \mathrm{m}$. Figures in the first column are BSE micrographs, those in the second are color-coded crystallite orientation maps, see colored stereographic triangle in the second column of (a) for color decoding. Figures in the third column are inverse pole figures (IPFs) showing the texture along the longitudinal axis of the compression specimens. 


\subsection{Effects of Grain Size and Temperature on Yield Stress}

Four representative compression true stress-true plastic strain curves at RT for crystallite sizes between 13 and $120 \mu \mathrm{m}$ are shown in Figure 5a. The yield stress as well as the flow stress at a given plastic strain are increasing with decreasing crystallite size. The effect of temperature on stress-strain behavior for a grain size of $d=33 \mu \mathrm{m}(c=25 \mu \mathrm{m})$ is shown in Figure $5 \mathrm{~b}$. The yield stresses are found to strongly decrease with increasing temperature between 77 and $673 \mathrm{~K}$. However, yield and flow stresses are similar at 673 and $873 \mathrm{~K}$, indicating the beginning of an athermal plateau (see also later Figure 6). Interestingly, while stress-strain curves are smooth at low temperatures, serrations are observed for $T>673 \mathrm{~K}$ regardless of the mean grain size. At $673 \mathrm{~K}$ the serrations appear right after yielding and disappear after $0.4 \%$ strain. In contrast, while the beginning of the stress-strain curve is smooth at $873 \mathrm{~K}$ serrations are first observed at $0.4 \%$ and are visible until the end of the compression test. The unstable plastic flow observed at high temperatures ( $T \geq 673 \mathrm{~K}$ ) may be attributed to the segregation of substitutional elements at stacking faults bounded by Shockley partials (Suzuki effect [38]) and/or the formation of Cottrell atmospheres [39]. Further work is still needed to clarify the nature of serrated flow occurring above $673 \mathrm{~K}$.

The effect of grain size on yield stress $\sigma_{y}$ can be described by the Hall-Petch relationship [40,41]:

$$
\sigma_{y}=\sigma_{0}+k_{\mathrm{y}} d^{-1 / 2}
$$

In Equation (1), two components contribute to the yield stress. The first component is the intrinsic lattice strength $\left(\sigma_{0}\right)$, which is representative of a polycrystalline material with an infinite crystallite size. The second term is the grain-size-dependent Hall-Petch contribution. From the mechanical tests performed in the present study, Hall-Petch parameters $\left(\sigma_{0}\right.$ and $\left.k_{y}\right)$ were determined by plotting the yield stress versus reciprocal square root of grain size $d$ or crystallite size $c$. A straight line with slope $k_{y}$ and $y$-intercept $\sigma_{0}$ was then adjusted to the data using a weighted least squares method. For a more detailed description of curve fitting as well as the assessment of uncertainties on fitted values ( $k_{y}$ and $\left.\sigma_{0}\right)$, see [21].

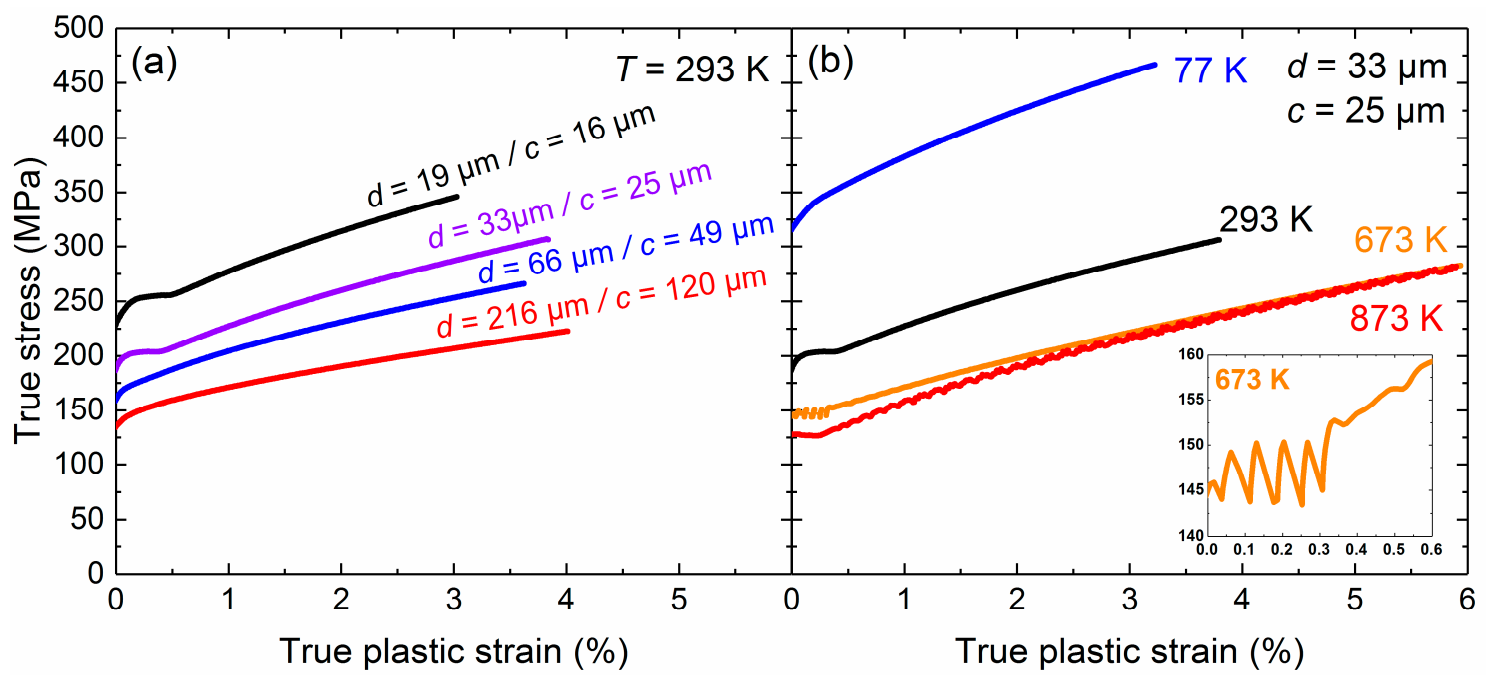

Figure 5. Representative true stress-true plastic strain curves obtained in compression of MnFeNi at (a) room temperature with different grain and crystallite sizes and (b) for a grain and crystallite size of 33 and $14 \mu \mathrm{m}$, respectively at different temperatures. The inset in (b) shows a serrated flow after yielding for the curve obtained at $673 \mathrm{~K}$. 


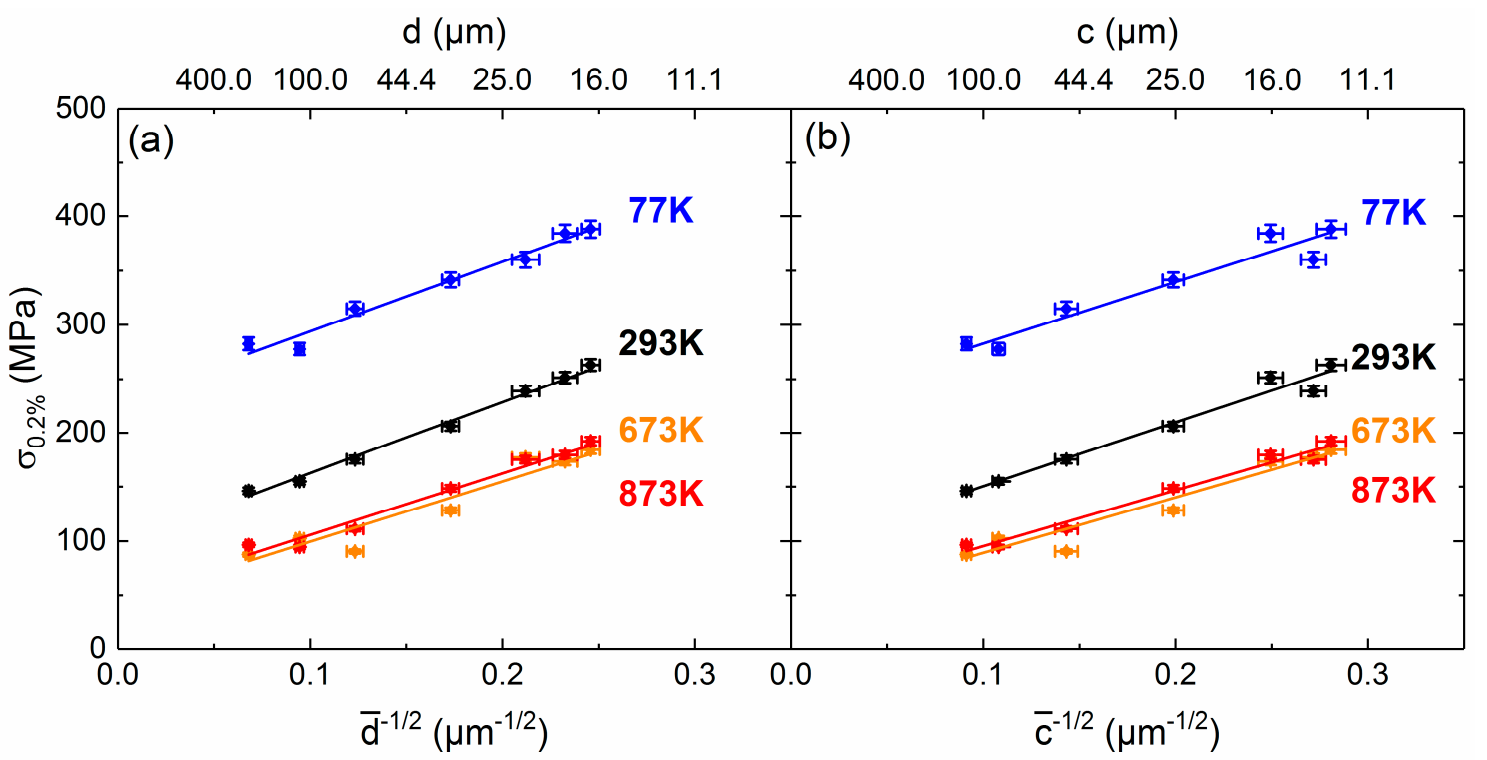

Figure 6. Hall-Petch plots at different temperatures. Yield stress $\left(\sigma_{0.2 \%}\right)$ versus (a) reciprocal of the square root of the grain size $(d)$ and $(\mathbf{b})$ crystallite size $(c)$.

The yield strength at $0.2 \%$ plastic strain is plotted as a function of $d^{-1 / 2}$ in Figure 6 a and $c^{-1 / 2}$ in Figure $6 \mathrm{~b}$ for temperatures ranging from 77 to $873 \mathrm{~K}$. At all investigated temperatures, the yield strength increases linearly with either $d^{-1 / 2}$ or $c^{-1 / 2}$ in good agreement with the Hall-Petch relationship (Equation (1)). The Hall-Petch parameters determined by fitting Equation (1) to the experimental data are given in Table 2 . Figure $6 \mathrm{a}, \mathrm{b}$ shows that the Hall-Petch lines are nearly parallel and that the intrinsic lattice strength $\sigma_{0}$ (y-intercept) is nearly constant between 673 and $873 \mathrm{~K}$ and strongly increases with decreasing temperature for $T<673 \mathrm{~K}$. A comparison of the Hall-Petch slopes in Table 2 reveals that those which account for both grain and twin boundaries (Figure $6 \mathrm{~b}$ ) are between 0.85 and 0.9 of those in Figure 6a. This result can be attributed to the fact that, for all grain sizes investigated in the present study, there is about one annealing twin boundary in one out of three grains (Figure 3a), i.e., $d \approx 1.33 c$. As a result, the slope is expected to decrease by a factor $1.33^{-1 / 2}=0.87$ (see Equation (1)) in perfect agreement with the results shown in Figure 6.

Table 2. Hall-Petch parameters $\left(\sigma_{0}\right.$ and $\left.k_{y}\right)$ determined by fitting Equation (1) to the grain size ( $d$, upper part) and crystallite size ( $c$, lower part) data sets obtained at different temperatures.

\begin{tabular}{|c|c|c|c|}
\hline Grain/Crystallite Sizes & $T(\mathrm{~K})$ & $\sigma_{0}(\mathrm{MPa})$ & $k_{y}\left(\mathrm{MPa} \mu \mathrm{m}^{1 / 2}\right)$ \\
\hline \multirow{4}{*}{$d$} & 77 & $230 \pm 7$ & $640 \pm 40$ \\
\hline & 293 & $97 \pm 4$ & $660 \pm 30$ \\
\hline & 673 & $44 \pm 3$ & $550 \pm 20$ \\
\hline & 873 & $49 \pm 3$ & $570 \pm 20$ \\
\hline \multirow{4}{*}{$c$} & 77 & $226 \pm 7$ & $570 \pm 40$ \\
\hline & 293 & $91 \pm 4$ & $590 \pm 25$ \\
\hline & 673 & $38 \pm 3$ & $510 \pm 20$ \\
\hline & 873 & $44 \pm 3$ & $515 \pm 20$ \\
\hline
\end{tabular}




\subsection{Temperature Dependence of the Yield Stress}

Figure 7 shows the temperature dependence of the $0.2 \%$ yield stress for the biggest crystallite size $(c=120 \mu \mathrm{m})$. Please note that four additional compression experiments were performed at 173, 223, 373 , and $473 \mathrm{~K}$. Two experiments were conducted at each temperature to provide the error bars. The yield stress for $d=216 \mu \mathrm{m}(c=120 \mu \mathrm{m})$, is found to strongly decrease with increasing temperature in the range $77-473 \mathrm{~K}$ while it remains nearly constant at higher temperatures suggesting that this corresponds to the athermal regime. In the former temperature region, the yield stress decreases by $\sim 65 \%$ while the shear modulus $(G)$ softens by $\sim 15 \%$ between 77 and $473 \mathrm{~K}$ [15]. Therefore, the large decrease of yield stress with temperature cannot be attributed only to a softening of the shear modulus, rather it suggests that plastic deformation is thermally activated in MnFeNi.

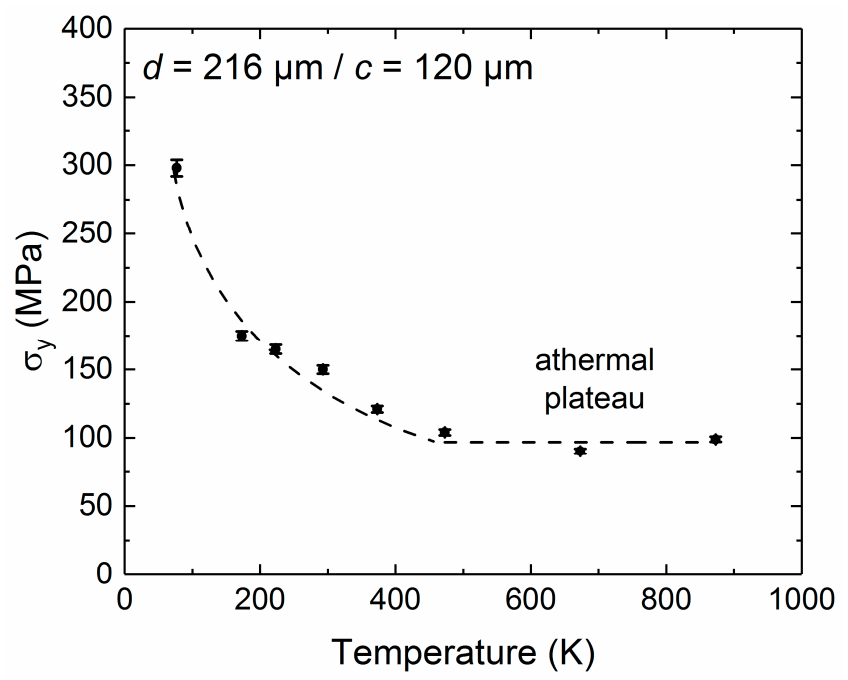

Figure 7. Temperature dependence of the yield strength $\left(\sigma_{y}\right)$ obtained for the largest grain size $(d=216 \mu \mathrm{m}, c=120 \mu \mathrm{m})$.

\subsection{STEM Analyses of Grain and Annealing Twin Boundaries}

To shed light on the interactions between dislocations and annealing twin and grain boundaries, STEM investigations were performed on a specimen which was strained to $1.5 \%$ plastic strain at $293 \mathrm{~K}$. In Figure 8a, three grain boundaries can be seen, meeting at a triple point. In the right grain, pile-ups containing large numbers of dislocations are observed, indicating that grain boundaries are effective obstacles to dislocation motion. Figure $8 \mathrm{~b}$ highlights similar features at a higher magnification where several pile-ups forming against grain boundaries are highlighted with red arrows. Figure $8 \mathrm{c}$ shows almost a complete grain in which one annealing twin boundary is present. In contrast, Figure $8 \mathrm{~d}$ from another grain shows two annealing twin boundaries at a higher magnification. In both cases (Figure $8 c, d$ ), dislocations are also found to pile-up against annealing twin boundaries. However, a comparison of Figure $8 \mathrm{~b}, \mathrm{~d}$ reveals that there are more dislocations in the pile-up at grain boundaries (see red arrows in Figure 8b) than at annealing twin boundaries (see white arrows in Figure 8d). This may indicate that annealing twin boundaries in MnFeNi are weaker obstacles to dislocation glide than grain boundaries. 

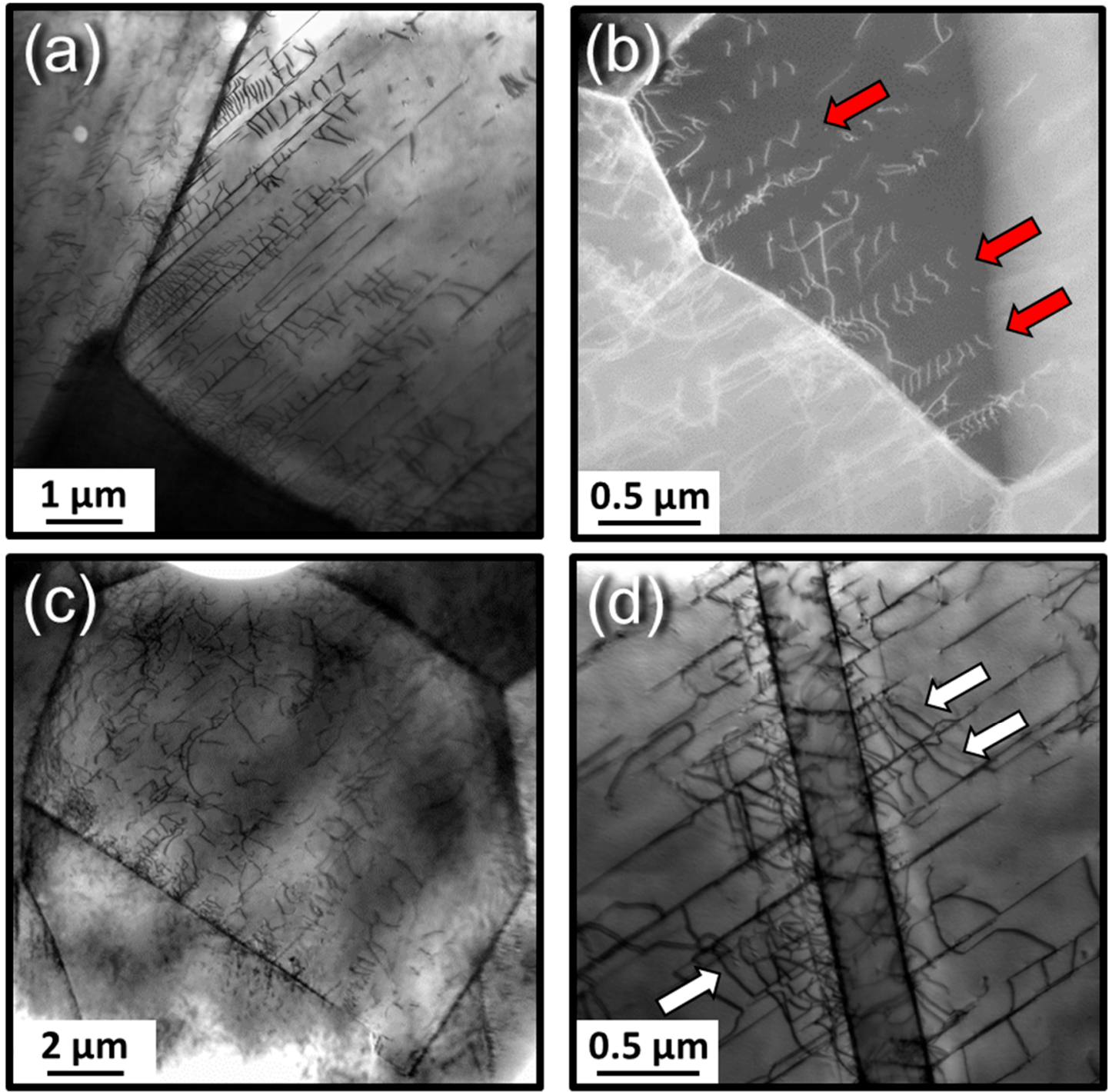

Figure 8. Scanning transmission electron microscopy (STEM) micrographs of a MnFeNi alloy $(d=17 \mu \mathrm{m})$ deformed to $1.5 \%$ true plastic strain at $293 \mathrm{~K}$ showing dislocations piling-up against $(\mathbf{a}, \mathbf{b})$ grain boundaries and $(\mathbf{c}, \mathbf{d})$ annealing twin boundaries $(\Sigma 3)$. Pile-ups of dislocations against grain boundaries and annealing twin boundaries are highlighted with red and white arrows, respectively.

\section{Discussion}

The objective of the present study was to investigate the effect of temperature and texture on grain size hardening (Hall-Petch relationship) in an equiatomic MnFeNi alloy. For this purpose, seven different grain sizes were produced by thermo-mechanical treatments, the corresponding textures were analyzed, and compression tests were performed between 77 and $873 \mathrm{~K}$. It was found that nearly no texture develops in the investigated grain size range 17-216 $\mu \mathrm{m}$ and that the annealing twin density in this alloy is relatively low. Furthermore, it was shown, that the grain size dependence of the yield stress follows the well-known Hall-Petch relationship [40,41], i.e., the yield stress decreases with increasing grain size. Finally, TEM observations revealed the presence of dislocation pile-ups against both grain and annealing twin boundaries. We now compare our data to those reported in the literature and discuss them with a focus on microstructural and mechanical aspects as well as their relationship.

In Figure 9, the average thickness of annealing twins $(t)$ determined using the line intercept method is plotted as a function of the mean grain size (d) for the MnFeNi alloy investigated in the present study (black data points). In addition, the data reported in the literature for CrCoNi [21] (red data points) and pure Ni [42] (blue data points) are shown for comparison in Figure 9. In all 
cases, the average thickness of the twins is found to increase linearly with increasing grain size in excellent agreement with the model developed by Dash and Brown [43] to describe the growth of annealing twins driven by the net reduction in grain boundary energy associated with twinning. More importantly, the slopes of the lines in Figure 9 should be proportional to the stacking fault energy according to the Dash and Brown model [43]. Therefore, based on the slopes shown in Figure 9 we expect the stacking fault energies of the metals to increase in the order:

$$
\text { CrCoNi (slope: } 0.13 \pm 0.01)<\mathrm{MnFeNi} \text { (slope: } 0.25 \pm 0.02)<\mathrm{Ni} \text { (slope: } 0.32 \pm 0.10 \text { ) }
$$

This trend is consistent with the SFEs of $\mathrm{CrCoNi}$ and pure Ni, which were reported to be $22 \pm 4 \mathrm{~mJ} / \mathrm{m}^{2}$ [44] and $125 \pm 4 \mathrm{~mJ} / \mathrm{m}^{2}$ [45], respectively. To our knowledge the SFE of MnFeNi has not been reported in the literature so far but it can be reasonably stated, based on the results shown in Figure 9, that its SFE should be closer to that of Ni than that of CrCoNi. If we assume that the Dash and Brown model applies and that the driving force for the formation of annealing twins is similar in all three alloys, then a linear interpolation of the SFE of MnFeNi yields an SFE-value of $80 \pm 20 \mathrm{~mJ} / \mathrm{m}^{2}$. However, further work is still required to confirm or disprove this value.

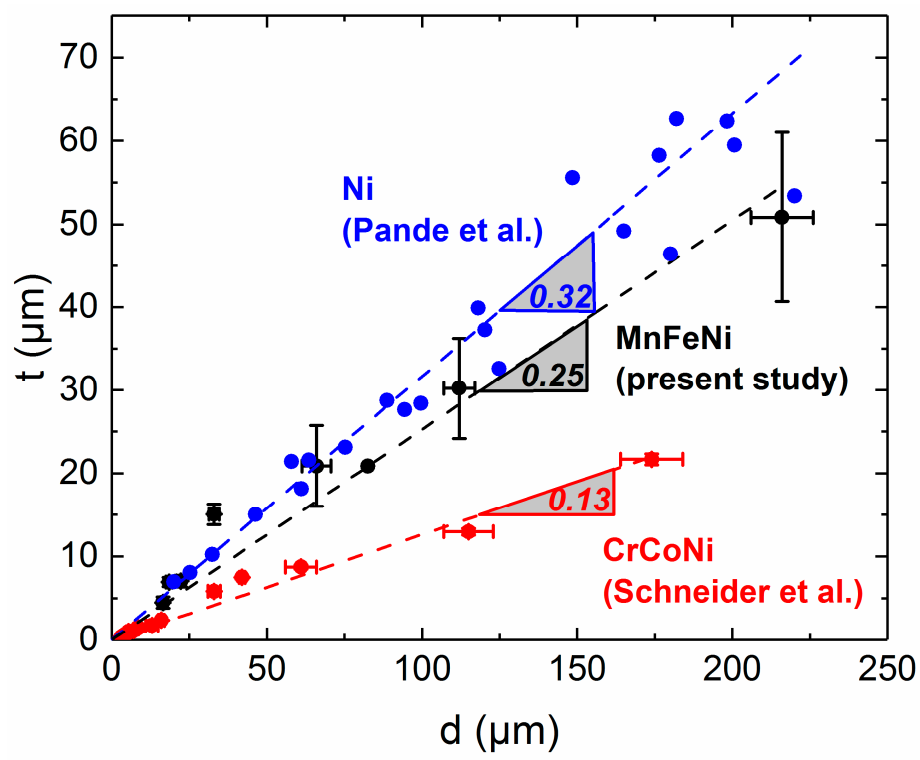

Figure 9. Average annealing twin thickness $(t)$ as a function of the mean grain size $(d)$. The black data points were obtained for $\mathrm{MnFeNi}$ in the present study, whereas the blue points were obtained from Pande et al. [42] for pure $\mathrm{Ni}$ and the red ones by Schneider et al. [21] for an equiatomic CrCoNi alloy.

The effect of temperature on Hall-Petch slope ( $k_{y}$, obtained from Figure 6$)$ is shown in Figure 10a. As already mentioned in Section 3.3, the magnitude of $k_{y}$, which reflects the strength of the boundaries, is larger when twin boundaries were excluded from the analysis (black data points, all boundary strengthening is attributed to grain boundaries) while it is slightly lower ( $10 \%$ to $15 \%$ ) when twin boundaries were accounted for (white data points, twin and grain boundaries are assumed to have the same strength in this case). Despite some scatter, a clear decrease of $k_{y}(\sim 15 \%)$ with increasing temperature is observed for both grain size (red line) and crystallite size data (blue line). 

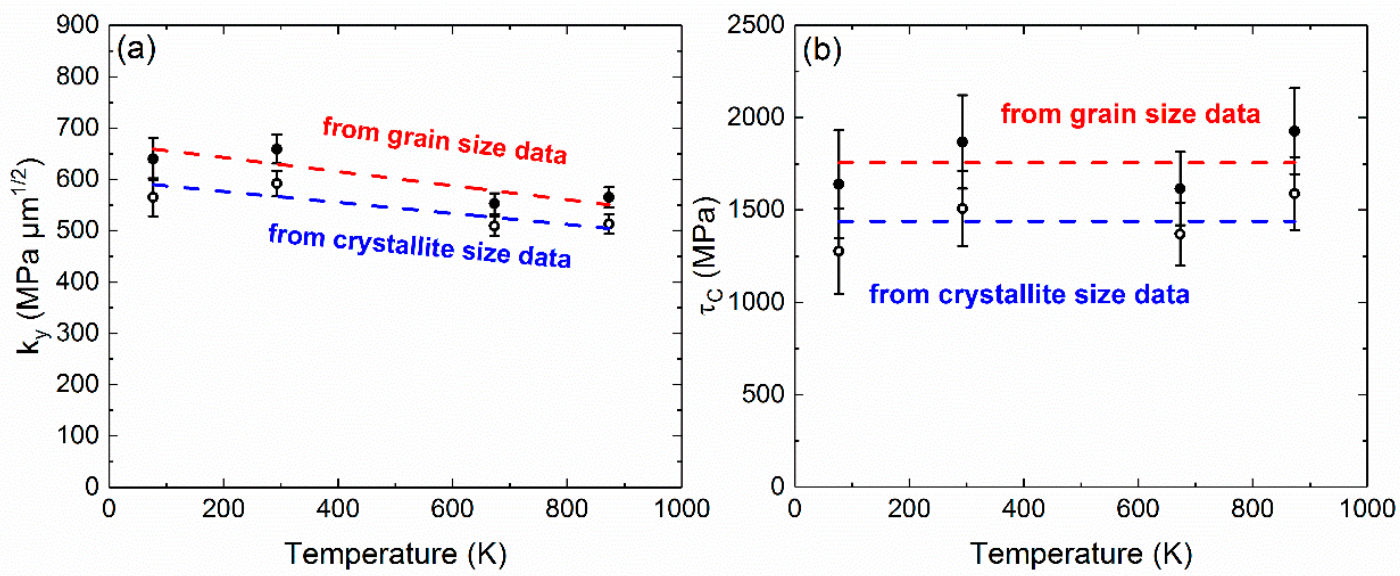

Figure 10. Temperature dependence of (a) Hall-Petch slope $\left(k_{y}\right)$ obtained from grain size data (red) and crystallite size data (blue) and (b) critical boundary strength $\left(\tau_{c}\right)$ obtained from grain sizes (filled points) and crystallite sizes (white points) assuming a pile-up of screw dislocations.

Microstructural observations by TEM of a compression sample pre-strained at $293 \mathrm{~K}$ revealed the presence of dislocation pile-ups against both grain and annealing twin boundaries suggesting that the dislocation pile-up model developed by Eshelby, Frank and Nabarro [46] is a reasonable rational for the Hall-Petch behavior. According to this model, the Hall-Petch slope $\left(k_{y}\right)$ is related to the boundary strength $\left(\tau_{c}\right.$, grain and/or twin boundary) which is required to continue slip past the boundary. In the case of a double ended pile-up of dislocations, these parameters are given by [27]:

$$
k_{\mathrm{y}}=M\left(\frac{\tau_{c} 4 \mathrm{G} b}{\beta \pi}\right)^{\frac{1}{2}}
$$

and

$$
\tau_{c}=\frac{k_{y}^{2} \beta \pi}{4 M^{2} G b^{2}}
$$

where $\beta$ is a factor taking into account the type of dislocation which is $\beta=1$ for screw and $\beta=1-v$ for edge dislocations, $M=3.06$ is the Taylor factor of an isotropic polycrystal, $b=0.256 \mathrm{~nm}$ is the Burgers vector of a perfect dislocation, $G$ is the shear modulus [15]. Here the Poisson's ratio at RT is $v=0.15$ and its temperature dependence was reported in [15]. Figure $10 \mathrm{~b}$ shows $\tau_{c}$ as a function of temperature for a pile-up of screw dislocations $(\beta=1)$. In spite of some scatter, the boundary strength is found to be nearly independent of temperature (see Figure 10b) for the grain size data (red line) as well as the crystallite size data (blue line).

In the present work, two outer bounds were used to assess boundary strengths. In the first case, it was assumed that annealing twins are very weak barriers to dislocation motion and can therefore be excluded. In this scenario, only grain boundaries act as barriers and their strength was determined using Equation (3) and the $k_{y}$ values shown Figure 10a. Assuming a pile-up of screw dislocations $(\beta=1)$ it is found that the grain boundary strength is $1750 \mathrm{MPa}$ when twin boundaries are excluded. In the second case, grain and twin boundaries are assumed to have the same strength resulting in a boundary strength of $1430 \mathrm{MPa}$. The latter value (1430 MPa) is $20 \%$ lower than that considering only grain boundaries. This value is consistent with the fact that $25 \%$ of the boundaries are twin boundaries (fraction of twin boundaries $\left.=(1 / n+1)^{-1}\right)$. For comparison the boundary strength values for pile-ups of edge dislocations $(\beta=1-v)$ are found to be $1430 \mathrm{MPa}$ for the former and $1180 \mathrm{MPa}$ for the latter case, respectively, which are $\sim 20 \%$ lower than in the case of pile-ups of screw dislocations $(\beta=1)$.

We now compare in Figure 11a our data (green points) to those (red points) reported by Wu et al. [9] for MnFeNi with a mean grain size of $\sim 30 \mu \mathrm{m}$. A comparison of the red and green data points reveals an excellent agreement at 77 and $293 \mathrm{~K}$ but the two sets of data deviates from each other's 
at higher temperatures. The data obtained by Wu et al. [9] also show an athermal plateau, similar to our data, but it lies $\sim 50 \mathrm{MPa}$ above our data. As already mentioned in Section 3.3, the strength of the MnFeNi medium-entropy alloy investigated in the present study consists of two contributions, namely, boundary strengthening and intrinsic lattice strength. To reveal the latter, boundary strengthening was removed by extrapolating the Hall-Petch lines in Figure 6 to an infinite grain size (low $d^{-1 / 2}$ ). Using the temperature dependence of the yield stress obtained for the biggest crystallite size and assuming a constant boundary strength, the intrinsic lattice strength was calculated for 173, 223, 373, and $473 \mathrm{~K}$ using Equations (1) and (2). The temperature dependence of $\sigma_{0}$ is shown in Figure $11 \mathrm{~b}$ based on either the analysis excluding twin boundaries (black symbols) or accounting for twin boundaries (empty symbols). The intrinsic lattice strength strongly decreases with increasing temperature between 77 and $473 \mathrm{~K}$, while it remains nearly constant between 473 and $873 \mathrm{~K}$ suggesting that this corresponds to the athermal regime. Recently, Varvenne et al. [47] have introduced a parameter-free theory for the temperature, composition, and strain-rate-dependence of the yield strength in FCC HEAs and MEAs. In this theory, each element is considered as a solute embedded in an effective matrix. Solid solution strengthening results from elastic interactions between dislocations and the random local concentration fluctuations around the mean composition. The inputs of this theory are the shear modulus, the Poisson ratio of the alloy, the dislocation Burgers vector $(b)$, the dislocation line tension, the individual atomic volumes, and atomic fractions of each element in the FCC alloy. The theory allows to calculate the temperature dependence of the yield stress (for examples see [21,47-49]) of the MnFeNi which is represented by a black line in Figure 11b. The theory underpredicts the yield stress at $77 \mathrm{~K}$ and is not accounting for the athermal plateau starting at $473 \mathrm{~K}$ but otherwise it is in generally good agreement with our experimental results for temperatures between 173 and $473 \mathrm{~K}$. Possible reasons for the differences observed at $77 \mathrm{~K}$ have been discussed by Varvenne et al. [47]. For example, the predictions are particularly sensitive to the line tension parameter. Moreover, short range ordering which was neglected in their model may be a reason for the discrepancy.

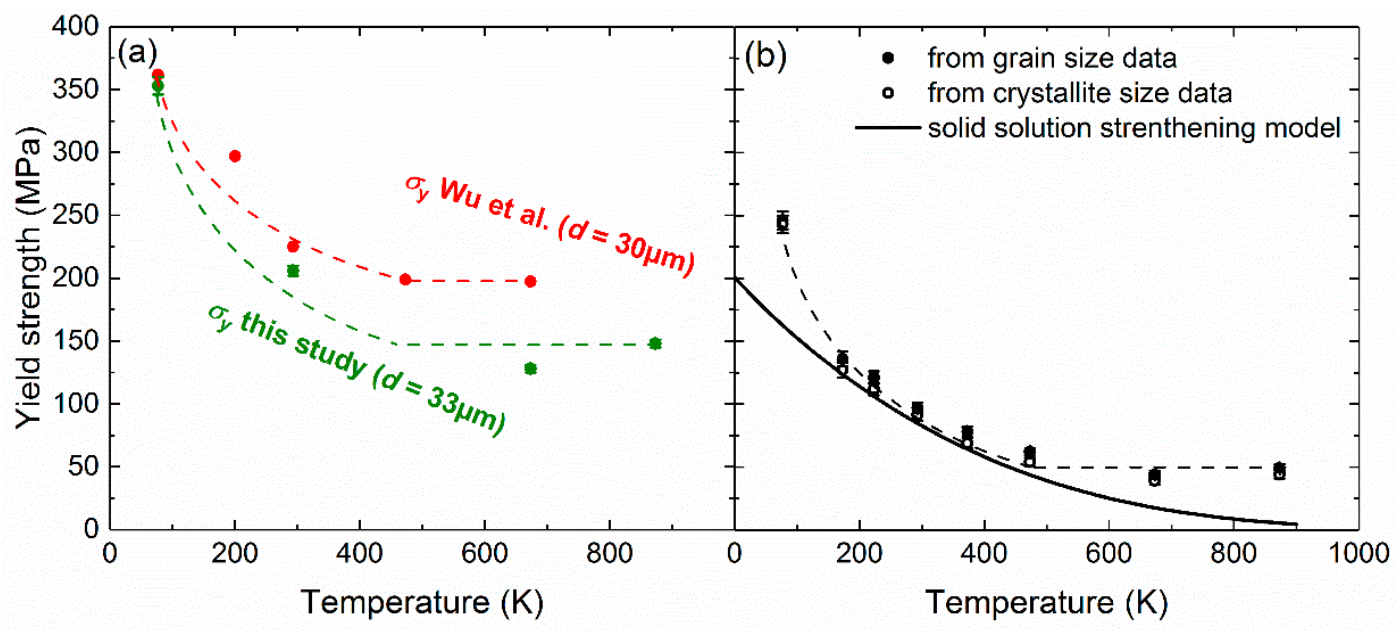

Figure 11. Temperature dependence of (a) yield strength obtained by Wu et al. [9] (red points) for a grain size of $30 \mu \mathrm{m}$ compared to values obtained in the present study for a grain size of $33 \mu \mathrm{m}$ (green points) and (b) intrinsic lattice strength of a polycrystal with an infinite grain/crystallite size. Black data points were obtained from Figure 6a by extrapolating the Hall-Petch plots to an infinite grain size while the empty symbols were determined by extrapolating the plots to an infinite crystallite size (Figure $6 b$ ). Also shown in (b) is the temperature dependence of the intrinsic lattice strength (black line) computed using the solid solution strengthening model of Varvenne et al. [47].

To illustrate the degree of solid solution strengthening of the MnFeNi alloy, the temperature dependence of its intrinsic lattice strength (black points) is compared with that of pure $\mathrm{Ni}$ (blue points) [50] and CrCoNi (red points) [21] in Figure 12. It can be clearly seen, that both MEAs alloys display a strong decrease of intrinsic lattice strength with increasing temperature. This is in contrast 
with pure Ni (blue points) which exhibits a very weak decrease with increasing temperature from $20 \mathrm{MPa}$ at $77 \mathrm{~K}$ to $15 \mathrm{MPa}$ at $623 \mathrm{~K}$. This may be attributed to the fact that solid solution strengthening does not contribute to its intrinsic lattice strength, which only consists of the Peierls-Nabarro stress. At a temperature of $77 \mathrm{~K}$, the strength of the $\mathrm{CrCoNi}$ alloy is much higher than that of the MnFeNi alloy $(\sim 40 \%)$ but this difference gradually decreases with increasing temperature until both curves merge at the athermal plateau in excellent agreement with results obtained by Wu et al. [9]. At $T>673 \mathrm{~K}$, both alloys exhibit roughly the same intrinsic lattice strength ( $50 \mathrm{MPa})$. From Figure 12 it can be seen that $\mathrm{CrCoNi}$ exhibits a higher yield strength in the range 77-673 K than MnFeNi, suggesting that different combinations of elements results in different magnitudes of solid solution strengthening, i.e., a combination of $\mathrm{Cr}$ and $\mathrm{Co}$ has a stronger effect on solid solution strengthening of $\mathrm{Ni}$ than a combination of Mn and Fe.

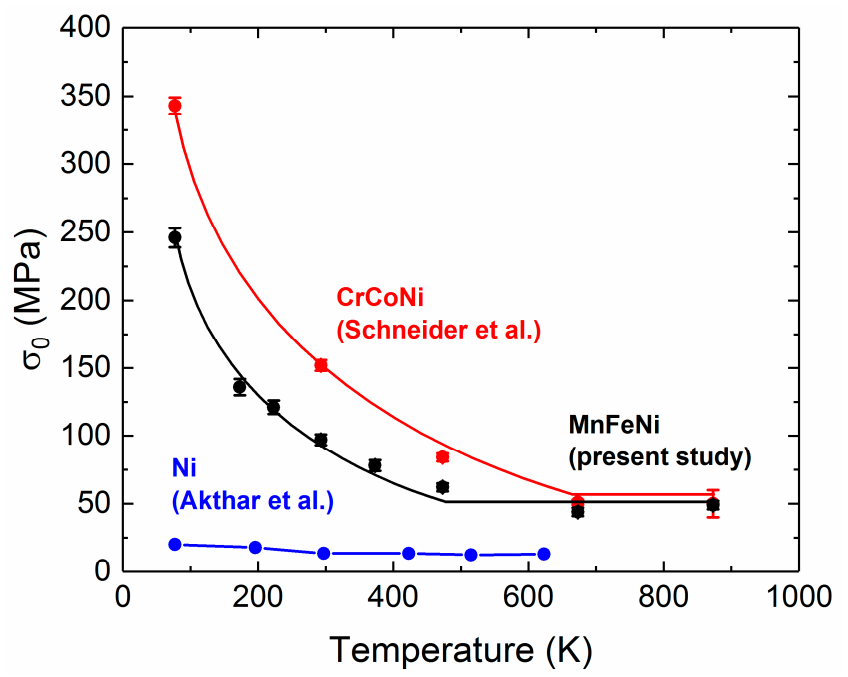

Figure 12. Yield strength $\left(\sigma_{0}\right)$ of single crystalline $\mathrm{Ni}$ [50] and polycrystalline $\mathrm{CrCoNi}$ [21] and $\mathrm{MnFeNi}$ (present study) extrapolated to an infinite grain size represented in blue, red and black, respectively, as a function of temperature determined by extrapolation of the grain sizes.

\section{Conclusions}

In the present work the equiatomic MnFeNi medium-entropy alloy was investigated. Seven different grain sizes between 17 and $216 \mu \mathrm{m}$ were produced and the effects of texture and temperature in the range between 77 and $873 \mathrm{~K}$ on the mechanical properties were documented. From the results of this work the following conclusions can be drawn:

- For all investigated grain/crystallite sizes, it has been found that the average number of annealing twin boundaries per grain in $\mathrm{MnFeNi}$ is approximately $0.35 \pm 0.1$. This value is relatively low compared to other high- and medium-entropy alloys from the Cr-Mn-Fe-Co-Ni system.

- When two twin boundaries forming an annealing twin are present in one grain, its average thickness is found to increase linearly with increasing grain size with a slope of $0.25 \pm 0.2$. Based on the model developed by Dash and Brown [43], we estimated a stacking fault energy of $80 \pm 20 \mathrm{~mJ} / \mathrm{m}^{2}$ for MnFeNi.

- The strength of the MnFeNi alloy follows the well-known Hall-Petch relationship for both grain and crystallite sizes, i.e., it increases with decreasing grain/crystallite sizes. Regarding the effect of temperature on the slope of the Hall-Petch lines, it was found that it slightly decreases $(\sim 15 \%)$ with increasing temperature in the range between 77 and $873 \mathrm{~K}$.

- The yield strength of a polycrystal with an infinite grain size ( single crystal behavior) strongly decreases with increasing temperature. This temperature dependence can be rationalized in the frame of a solid solution strengthening model reported in the literature [47] for $173 \mathrm{~K}<T<473 \mathrm{~K}$. Above $473 \mathrm{~K}$, an athermal plateau is reached. 
- STEM analyses reveal that pile-ups form at both grain and annealing twin boundaries. However, pile-ups against annealing twins seems to be shorter than against grain boundaries which may indicate that annealing twins are less resistant than grain boundaries against dislocation motion.

Author Contributions: M.S. and G.L. conceived and designed the experiments; C.R. prepared the alloy and performed the preliminary heat treatments. F.W. and M.S. performed the microstructural and mechanical investigations and analyzed the data; D.L. prepared the TEM specimens and performed the corresponding experiments; F.W., M.S., D.L. and G.L. wrote the paper together. G.L. supervised the research activity planning and execution. All authors contributed substantially to the work reported.

Funding: M.S., C.R., and G.L. acknowledge funding from the German Research Foundation (Deutsche Forschungsgemeinschaft DFG) through project LA $3607 / 1-1$ and projects B5 and B7 of the SFB/TR 103. The center for interface-dominated high performance materials (Zentrum für Grenzflächendominierte Höchstleistungswerkstoffe, ZGH) is acknowledged.

Acknowledgments: The authors would like to thank Gernot Hausch and Easo P. George for fruitful discussions.

Conflicts of Interest: The authors declare no conflict of interest. The funders had no role in the design of the study; in the collection, analyses, or interpretation of data; in the writing of the manuscript, or in the decision to publish the results.

\section{References}

1. Yeh, J.-W.; Chen, S.-K.; Lin, S.-J.; Gan, J.-Y.; Chin, T.-S.; Shun, T.-T.; Tsau, C.-H.; Chang, S.-Y. Nanostructured high-entropy alloys with multiple principal elements: Novel alloy design concepts and outcomes. Adv. Eng. Mater. 2004, 6, 299-303. [CrossRef]

2. Yeh, J.-W.; Chen, Y.-L.; Lin, S.-J.; Chen, S.-K. High-entropy Alloys-A New Era of Exploitation. Mater. Sci. Forum 2007, 560, 1-9. [CrossRef]

3. Cantor, B.; Chang, I.T.H.; Knight, P.; Vincent, A.J.B. Microstructural development in equiatomic multicomponent alloys. Mater. Sci. Eng. A 2004, 375-377, 213-218. [CrossRef]

4. Laurent-Brocq, M.; Akhatova, A.; Perrière, L.; Chebini, S.; Sauvage, X.; Leroy, E.; Champion, Y. Insights into the phase diagram of the CrMnFeCoNi high entropy alloy. Acta Mater. 2015, 88, 355-365. [CrossRef]

5. Senkov, O.N.; Wilks, G.B.; Scott, J.M.; Miracle, D.B. Mechanical properties of $\mathrm{Nb}_{25} \mathrm{Mo}_{25} \mathrm{Ta}_{25} \mathrm{~W}_{25}$ and $\mathrm{V}_{20} \mathrm{Nb}_{20} \mathrm{Mo}_{20} \mathrm{Ta}_{20} \mathrm{~W}_{20}$ refractory high entropy alloys. Intermetallics 2011, 19, 698-706. [CrossRef]

6. Couzinié, J.P.; Dirras, G.; Perrière, L.; Chauveau, T.; Leroy, E.; Champion, Y.; Guillot, I. Microstructure of a near-equimolar refractory high-entropy alloy. Mater. Lett. 2014, 126, 285-287. [CrossRef]

7. Dobbelstein, H.; Gurevich, E.L.; George, E.P.; Ostendorf, A.; Laplanche, G. Laser metal deposition of a refractory TiZrNbHfTa high-entropy alloy. Addit. Manuf. 2018, 24, 386-390. [CrossRef]

8. Gali, A.; George, E.P. Tensile properties of high- and medium-entropy alloys. Intermetallics 2013, 39 , 74-78. [CrossRef]

9. Wu, Z.; Bei, H.; Pharr, G.M.; George, E.P. Temperature dependence of the mechanical properties of equiatomic solid solution alloys with face-centered cubic crystal structures. Acta Mater. 2014, 81, 428-441. [CrossRef]

10. Otto, F.; Dlouhý, A.; Somsen, C.; Bei, H.; Eggeler, G.; George, E.P. The influences of temperature and microstructure on the tensile properties of a CoCrFeMnNi high-entropy alloy. Acta Mater. 2013, 61, 5743-5755. [CrossRef]

11. Laplanche, G.; Kostka, A.; Horst, O.M.; Eggeler, G.; George, E.P. Microstructure evolution and critical stress for twinning in the CrMnFeCoNi high-entropy alloy. Acta Mater. 2016, 118, 152-163. [CrossRef]

12. Gludovatz, B.; Hohenwarter, A.; Catoor, D.; Chang, E.H.; George, E.P.; Ritchie, R.O. A fracture-resistant high-entropy alloy for cryogenic applications. Science 2014, 345, 1153-1158. [CrossRef] [PubMed]

13. Thurston, K.V.S.; Gludovatz, B.; Hohenwarter, A.; Laplanche, G.; George, E.P.; Ritchie, R.O. Effect of temperature on the fatigue-crack growth behavior of the high-entropy alloy CrMnFeCoNi. Intermetallics 2017, 88, 65-72. [CrossRef]

14. Wu, Z.; Bei, H.; Otto, F.; Pharr, G.M.; George, E.P. Recovery, recrystallization, grain growth and phase stability of a family of FCC-structured multi-component equiatomic solid solution alloys. Intermetallics 2014, 46, 131-140. [CrossRef] 
15. Laplanche, G.; Gadaud, P.; Bärsch, C.; Demtröder, K.; Reinhart, C.; Schreuer, J.; George, E.P. Elastic moduli and thermal expansion coefficients of medium-entropy subsystems of the CrMnFeCoNi high-entropy alloy. J. Alloys Compd. 2018, 746, 244-255. [CrossRef]

16. Shiga, M. Invar alloys. Curr. Opin. Solid State Mater. Sci. 1996, 1, 340-348. [CrossRef]

17. Hausch, G. Thermal expansion of anti-invar alloys up to high temperatures. J. Magn. Magn. Mater. 2007, 310, 1859-1860. [CrossRef]

18. Liu, W.H.; Wu, Y.; He, J.Y.; Nieh, T.G.; Lu, Z.P. Grain growth and the Hall-Petch relationship in a high-entropy FeCrNiCoMn alloy. Scr. Mater. 2013, 68, 526-529. [CrossRef]

19. Sun, S.J.; Tian, Y.Z.; Lin, H.R.; Dong, X.G.; Wang, Y.H.; Zhang, Z.J.; Zhang, Z.F. Enhanced strength and ductility of bulk CoCrFeMnNi high entropy alloy having fully recrystallized ultrafine-grained structure. Mater. Des. 2017, 133, 122-127. [CrossRef]

20. Yoshida, S.; Bhattacharjee, T.; Bai, Y.; Tsuji, N. Friction stress and Hall-Petch relationship in CoCrNi equiatomic medium entropy alloy processed by severe plastic deformation and subsequent annealing. Scr. Mater. 2017, 134, 33-36. [CrossRef]

21. Schneider, M.; George, E.P.; Manescau, T.J.; Záležák, T.; Hunfeld, J.; Dlouhý, A.; Eggeler, G.; Laplanche, G. Hall-Petch strengthening of the $\mathrm{CrCoNi}$ medium-entropy alloy by grain boundaries and annealing twin boundaries. manuscript in preparation.

22. Klimova, M.; Stepanov, N.; Shaysultanov, D.; Chernichenko, R.; Yurchenko, N.; Sanin, V.; Salishchev, G. Microstructure and Mechanical Properties Evolution of the Al, C-Containing CoCrFeNiMn-Type High-Entropy Alloy during Cold Rolling. Materials 2018, 11, 53. [CrossRef] [PubMed]

23. Stepanov, N.; Tikhonovsky, M.; Yurchenko, N.; Zyabkin, D.; Klimova, M.; Zherebtsov, S.; Efimov, A.; Salishchev, G. Effect of cryo-deformation on structure and properties of CoCrFeNiMn high-entropy alloy. Intermetallics 2015, 59, 8-17. [CrossRef]

24. Holcomb, G.; Tylczak, J.; Carney, C. Oxidation of CoCrFeMnNi High Entropy Alloys. JOM 2015, 67, 2326-2339. [CrossRef]

25. Laplanche, G.; Volkert, U.F.; Eggeler, G.; George, E.P. Oxidation Behavior of the CrMnFeCoNi High-Entropy Alloy. Oxid. Met. 2016, 85, 629-645. [CrossRef]

26. Adomako, N.K.; Kim, J.H.; Hyun, Y.T. High-temperature oxidation behaviour of low-entropy alloy to medium- and high-entropy alloys. J. Therm. Anal. Calorim. 2018, 133, 13-16. [CrossRef]

27. Dieter, G.E.; Bacon, D.J. Strengthening Mechanisms. Mechanical Metallurgy, 3rd ed.; McGraw-Hill: London, UK, 1989; ISBN 0-07-100406-8.

28. American Society for Testing and Materials (ASTM). ASTM E112-10: Standard Test Methods for Determining Average Grain Size; ASTM International: West Conshohocken, PA, USA, 2004.

29. Nakanishi, K.; Suzuki, H. Analysis of the Grain Size Dependence of the Yield Stress in Copper-Aluminum and Copper-Nickel Alloys. J. Jpn. Inst. Met. 1974, 15, 435-440. [CrossRef]

30. Ueji, R.; Tsuchida, N.; Fujii, H.; Kondo, D.; Kunishige, K. Effect of Grain Size on Tensile Properties of TWIP Steel. J. Jpn. Inst. Met. 2007, 71, 815-821. [CrossRef]

31. Okamoto, N.L.; Fujimoto, S.; Kambra, Y.; Kawamura, M.; Chen, Z.M.T.; Matsunoshita, H.; Tanaka, K.; Inui, H.; George, E.P. Size effect, critical resolved shear, stress, stacking fault energy, and solid solution strengthening in the CrMnFeCoNi high-entropy alloy. Sci. Rep. 2016, 6, 1-10. [CrossRef]

32. Köster, W.; Speidel, M.O. Der Einfluß der Temperatur und der Korngröße auf die ausgeprägte Streckgrenze von Kupferlegierungen. Zeitschrift für Metallkunde 1965, 56, 585-595. (In German)

33. Tabata, T.; Takagi, K.; Fujita, H. The Effect of Grain Size and Deformation Sub-structure on Mechanical Properties of Polycrystalline Copper and Cu-Al Alloys. J. Jpn. Inst. Met. 1975, 16, 569-579. [CrossRef]

34. Priester, L. "Dislocation-interface" interaction-Stress accommodation processes at interfaces. Mater. Sci. Eng. A 2001, 309-310, 430-439. [CrossRef]

35. Pande, C.S.; Rath, B.B.; Imam, M.A. Effect of annealing twins on Hall-Petch relation in polycrystalline materials. Mater. Sci. Eng. A 2004, 367, 171-175. [CrossRef]

36. De las Cuevas, F.; Reis, M.; Ferraiuolo, A.; Pratolongo, G.; Karjalainen, L.P.; Alkorta, J.; Sevillani, J.G. Hall-Petch relationship of a TWIP steel. Key Eng. Mater. 2010, 423, 147-152. [CrossRef]

37. Kacher, J.; Eftink, B.P.; Cui, B.; Robertson, I.M. Dislocation interactions with grain boundaries. Curr. Opin. Solid State Mater. Sci. 2017, 18, 227-243. [CrossRef] 
38. Suzuki, H. Chemical Interaction of Solute Atoms with Dislocations. Sci. Rep. Res. Inst. Tohoku Univ. 1952, 4, 455-463.

39. Cottrell, A.H.; Bilby, B.A. Dislocation theory of yielding and strain ageing of iron. Proc. Phys. Soc. Lond. Sect. A 1949, 62, 49-62. [CrossRef]

40. Hall, E.O. The Deformation and Ageing of Mild Steel: III Discussion of Results. Proc. Phys. Soc. Sect. B 1951, 64, 747-753. [CrossRef]

41. Petch, N.J. The cleavage strength of polycrystals. J. Iron Steel Inst. 1953, 174, 25-28.

42. Pande, C.S.; Imam, M.A.; Rath, B.B. Study of Annealing Twins in FCC Metals and Alloys. Metall. Trans. A 1990, 21, 2891-2896. [CrossRef]

43. Dash, S.; Brown, N. An investigation of the origin and growth of annealing twins. Acta Mater. 1963, 11, 1067-1075. [CrossRef]

44. Laplanche, G.; Kostka, A.; Reinhart, C.; Hunfeld, J.; Eggeler, G.; George, E.P. Reasons for the superior mechanical properties of medium-entropy $\mathrm{CrCoNi}$ compared to high-entropy CrMnFeCoNi. Acta Mater. 2017, 128, 292-303. [CrossRef]

45. Carter, C.B.; Holmes, S.M. The stacking-fault energy of nickel. Philos. Mag. J. Theor. Exp. Appl. Phys. 1977, 35, 1161-1172. [CrossRef]

46. Eshelby, J.D.; Frank, F.C.; Nabarro, F.R.N. XLI. The equilibrium of linear arrays of dislocations. Lond. Edinburgh Dublin Philos. Mag. J. Sci. 1951, 42, 351-364. [CrossRef]

47. Varvenne, C.; Luque, A.; Curtin, W.A. Theory of strengthening in fcc high entropy alloys. Acta Mater. 2016, 118, 164-176. [CrossRef]

48. Laplanche, G.; Bonneville, J.; Varvenne, C.; Curtin, W.A.; George, E.P. Thermal activation parameters of plastic flow reveal deformation mechanisms in the CrMnFeCoNi high-entropy alloy. Acta Mater. 2018, 143, 257-264. [CrossRef]

49. Varvenne, C.; Curtin, W.A. Predicting yield strengths of noble metal high entropy alloys. Scr. Mater. 2018, 142, 92-95. [CrossRef]

50. Akhtar, A.; Teghtsoonian, E. Plastic deformation of Ni-Cr single crystals. Metall. Trans. 1971, 2, $2757-2763$. [CrossRef]

(C) 2019 by the authors. Licensee MDPI, Basel, Switzerland. This article is an open access article distributed under the terms and conditions of the Creative Commons Attribution (CC BY) license (http://creativecommons.org/licenses/by/4.0/). 